\title{
Solving the enigma of posterolateral tibial plateau fractures, the clue protocol
}

\author{
Radwan G. Metwaly, Zeiad M. Zakaria, Mohamed A. Elgebeily, Hany El Zahlawy \\ From the Faculty of Medicine, Orthopedics Department, Ain Shams University, Cairo, Egypt
}

The study aim is to evaluate functional and radiological outcomes following a suggested protocol based on the four-column classification for management of posterolateral column tibial plateau fractures.

A prospective cohort study was performed in level I academic center on 42 patients with mean age of 36 years (22-59). Eleven patients had isolated posterolateral column fractures whereas 31 patients had associated columns fractures. According to the suggested protocol, all cases of isolated posterolateral column fracture started treatment via arthroscopic evaluation of soft tissue injuries (menisci and ligaments), arthroscopically assisted reduction and internal fixation by rafting screws followed by ORIF if plating was needed. If associated with other columns fractures, columns were fixed sequentially in an anti-clockwise direction starting from anteromedial column.

Average follow up was $\mathbf{2 6}$ months. Mean time to union was 16.3 (12-22) weeks. No radiological evidence of loss of coronal or sagittal alignment was detected at final follow up. Five patients had an average depression of 5 millimeters that did not need further intervention at this short-term follow up. Mean KOOS was 81 (72$88)$. The average knee range of motion was $\left(0^{\circ}-127^{\circ}\right)$. One patient had temporary common peroneal nerve injury, one patient had deep infection and two had superficial wound infection.

implementing the suggested protocol gives good to excellent radiological and functional results as regard posterolateral tibial plateau fracture. A larger study group with longer follow up is needed.

Keywords : Tibial plateau fractures; postero-lateral column ; protocol.

No benefits or funds were received in support of this study. None of the authors have a conflict of interest.

\section{INTRODUCTION}

Through the last decade, tibial plateau fractures have shown dramatic evolution in their diagnostic prerequisites together with new surgical approaches, reduction techniques and fixation implants being implemented $(1,2)$. This could be attributed to the three-column theory based on three-column CT classification presented by Luo et al (3).

Increased attention towards the coronal plane fractures as predictors of reduction loss increased the use of the posterior, posteromedial and posterolateral approaches (2-6). With further understanding of the column principle, better functional outcome and overall results have been recorded (2).

Posteromedial coronal fractures have previously been studied well in the literature (either isolated or in bicondylar plateau fractures) with substantial agreement on the necessity of using posteromedial approach with anti-shear buttress plating of these fragments to avoid postoperative collapse $(4,5,7)$.

Radwan G. Metwaly, AP

Zeiad M. Zakaria, PhD

Mohamed A. Elgebeily, PhD

- Hany El Zahlawy, PhD

Faculty of Medicine, Orthopedics Department, Ain Shams University, Cairo, Egypt.

Correspondence : Hany El Zahlawy, Address : 18 Rabaa Housing Project, El Nasr city, Cairo, Egypt. Phone: +201002508641, Fax : +20224509378.

Email : hzahlawy@hotmail.com

- 2021, Acta Orthopædica Belgica. 
On the other hand, the enigma of posterolateral tibial plateau fractures as regards their best fixation technique is still unsolved. Different new classifications have been introduced and many modifications of the posterolateral approach were described (8-11). Unfortunately, none of these previously described gates are completely innocent. Innovating more biological and minimally invasive techniques minimizes the complications associated with such extensive approaches. However, this should not be on the expense of biomechanical stability or increased risk of neurovascular compromise $(4,12,13)$.

Arthroscopically assisted reduction and internal fixation (ARIF) of tibial plateau fractures have shown good to excellent results even with long term follow ups (14). It allows direct visualization of the articular fracture surface with its more precise reduction in comparison to image intensifier alone, minimal soft tissue disruption and most importantly it can address the associated meniscal and ligamentous injuries especially the lateral meniscus tears, with their repair providing better functional outcomes $(15,16)$.

This study suggests a protocol based on precise diagnosis for managing posterolateral plateau fractures (either isolated or associated with other columns injuries). Choosing the proper approach, reduction technique and fixation implant facilitates the postoperative rehabilitation, gives better functional outcome and prevents the progression of osteoarthritis.

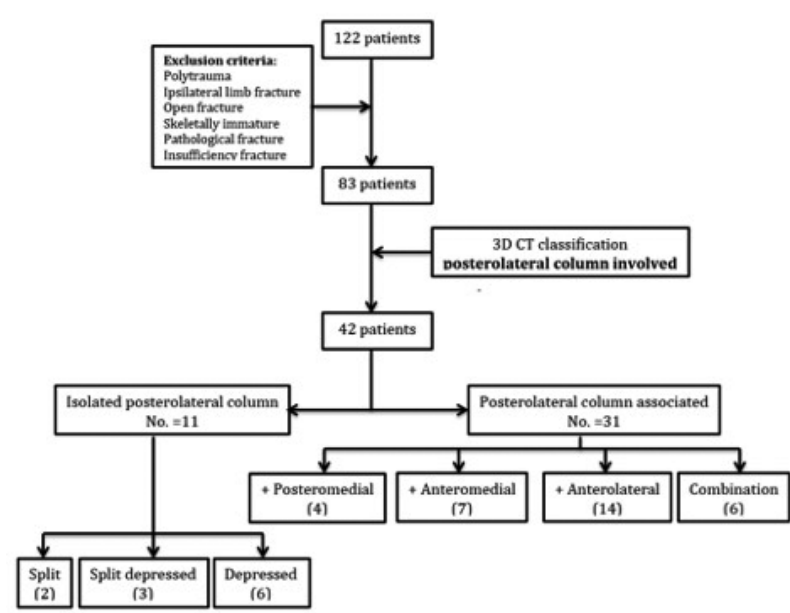

Fig 1. - Diagram of patient selection in methodology.

\section{PATIENTS AND METHODS}

Between June 2015 and December 2018, a prospective cohort clinical study was conducted in a level I academic center on patients with tibial plateau fractures. Informed consent was obtained from all individual participants included in the study. All procedures performed in this study were in accordance with the ethical standards of the institutional and national research committee. Polytraumatized patients, those with associated ipsilateral limb fractures, open fractures, pathological fractures including stress insufficiency fractures and skeletally immature patients were excluded from the study.

Table 1. - Number of patients in the study regarding mechanism of the trauma, different types and whether isolated or combined with other column(s) fracture

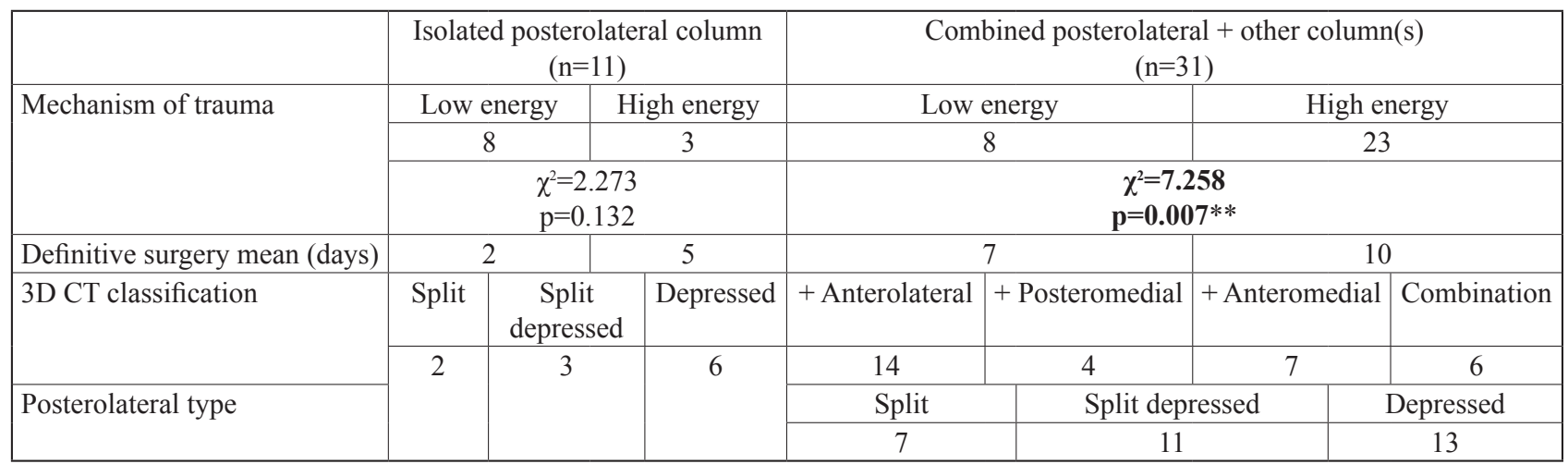

$* * \mathrm{p}<0.01$ : is highly significant. 
From 122 tibial plateau fractures being treated, 83 were included in this study. 3D-CT scan was done for the patients to analyze the fracture pattern. Based on the four-column classification proposed by Chang SM et al (17), 42 patients (34.4\%) had posterolateral column involvement; 11 with isolated column fracture while 31 had their posterolateral column fracture associated with other columns fractures (figure 1).

Mechanism of injury was recorded as either highenergy trauma (road traffic injury, fall from height more than three meters and heavy blunt object trauma) or low-energy trauma (fall from height less than three meters, sports injury or slip on ground). A staged procedure as opposed to a single staged surgery was performed for the high-energy injuries to optimize the soft tissue condition for better wound healing in 26 patients (table 1).

All patients were classified on initial X-rays according to Schatzker. If a staged procedure was proposed, 3D-CT scan was postponed till after initial reduction and traction with external fixation for better orientation of fracture fragments. The definitive fixation was postponed till improvement of soft tissue condition with a mean gap of 8 days between external and internal fixation. Four-column classification was used to plan the definitive surgical approach, reduction technique and implants needed for fracture fixation.

The basic principles for fixation of intra-articular fractures were applied in all cases to allow early range of motion. Anatomical reduction and rigid stability of articular surface was followed by alignment reduction and relative stability of metaphyseal fracture with or without bone grafting. The suggested protocol as shown in (figure 2) was used to plan the needed approaches and fixation implants. In details, the protocol we propose is as follows :

1. Posterolateral approach with posterior buttress plating with or without bone graft is done in :

- Isolated posterolateral plateau fracture, split or split depressed type with the height and width of the cortical fragment more than $15 \mathrm{~mm}$ (in order to have a surface area which accepts at least two screws in the horizontal limb of the plate).

- Isolated posterolateral plateau fracture, split or split depressed type with the height and

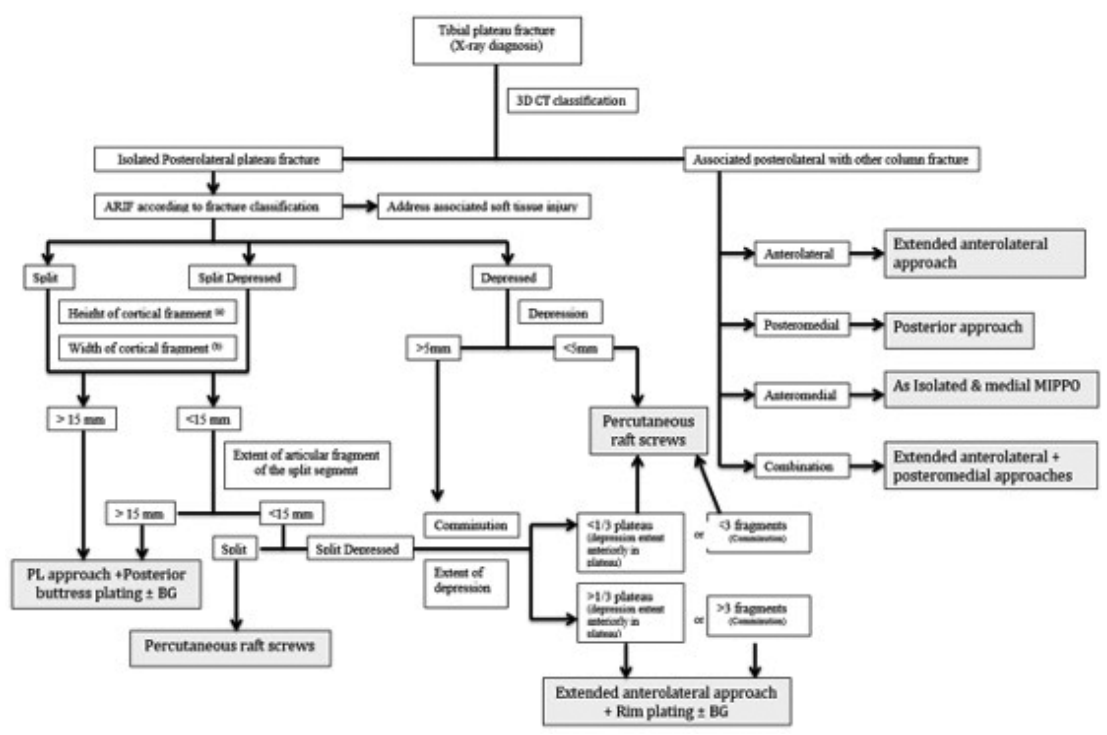

Fig 2. - The "clue" protocol suggested to solve the enigma of posterolateral tibial plateau fractures (a) the height is the vertical distance in the sagittal plane from the plateau surface till the distal extension of the cortical fragment (b) the width is the horizontal distance in the coronal plane (both criteria of height and width are needed for decision making).

$\mathrm{MIPPO}=$ minimally invasive percutaneous plate osteo-synthesis. $\mathrm{BG}=$ bone grafting. $\mathrm{PL}=$ posterolateral. ARIF $=$ arthroscopically assisted reduction and internal fixation. 
width of the cortical fragment less than $15 \mathrm{~mm}$ but the extent of articular fragment of the split segment more than $15 \mathrm{~mm}$.

2. Per cutaneous rafting screws is done in :

- Isolated posterolateral plateau fracture, split type with the height and width of the cortical fragment less than $15 \mathrm{~mm}$ and the extent of articular fragment of the split segment also less than $15 \mathrm{~mm}$.

- Isolated posterolateral plateau fracture, splitdepression type with the height and width of the cortical fragment less than $15 \mathrm{~mm}$ and the extent of articular fragment of the split segment also less than $15 \mathrm{~mm}$ provided there is no comminution (less than 3 fragments) and the extent of the depressed area in the plateau is less than its one third.

- Isolated posterolateral plateau fracture, depression type and the depression is less than $5 \mathrm{~mm}$.

- Isolated posterolateral plateau fracture, depression type and the depression is more than $5 \mathrm{~mm}$ but extent of depression is less than one third of the plateau or not comminuted (less than three fragments).

3. Extended anterolateral approach with posterior buttress plating with or without bone graft is done in the following conditions :

- Isolated posterolateral plateau fracture, split depressed or depression types and the depression is more than $5 \mathrm{~mm}$ but extent of depression is more than one third of the plateau or comminuted (more than three fragments)

- Associated posterolateral with anterolateral fractures

4. Extended anterolateral approach and posteromedial approach are done in multiple combinations of fracture patterns.

5. Posterior approach is done in posterolateral associated with posteromedial fractures or alternatively, we can use separate incisions : posteromedial and posterolateral approaches.

6. If anteromedial fracture is present in association with posterolateral fracture then they are managed as isolated posterolateral fracture and medial minimally invasive percutaneous plate osteosynthesis (MIPPO)

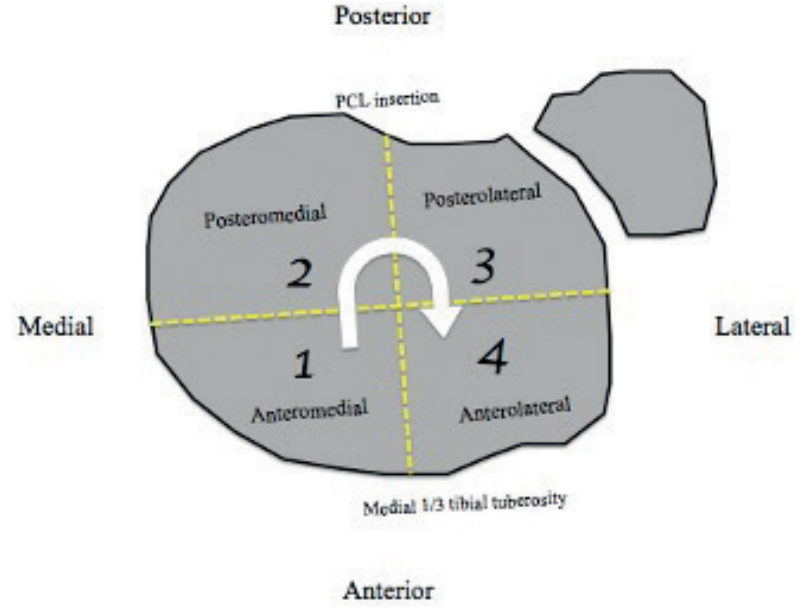

Fig 3. - The sequence of column fixation in associated columns fracture of tibial plateau starting from the anteromedial fragment.

7. Combined anterolateral and posterolateral columns fractures : Extended anterolateral approach is used and rim plating for posterolateral fragment fixation (considered as if isolated depression or split depression type with extent in the plateau more than one third)

When dealing with posterolateral column injury, sequence of fracture reduction and fixation was dictated by the diagnosis, whether it was isolated or combined injury. When combined with anteromedial column injury, anteromedial approach was utilized according to the protocol. The whole procedure was performed in the supine position unless additional posterior or posterolateral approaches were needed. Reduction of the anteromedial column was initially done to obtain a stable leveled plateau (the cornerstone of reduction) followed by posterolateral column fixation in the supine position when using rafting screws only. In cases needing buttress plating via the additional approaches, the patients were repositioned prone. In cases with combined anterolateral column affection, all the procedure was completed again in the supine position, however the reduction was performed from posterior to anterior using the extended anterolateral approach. With posteromedial column affection the procedure could be done either in supine position only using posteromedial approach or was completed in prone position when posterolateral plating was needed 


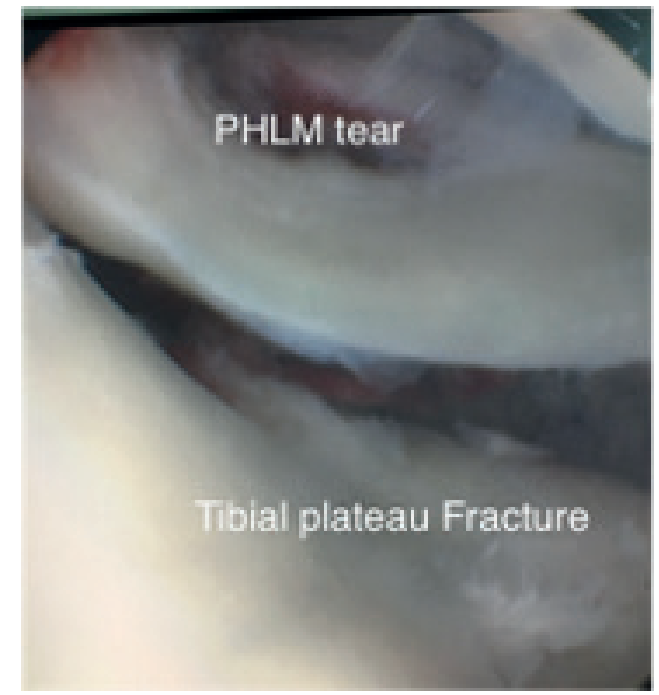

Fig 4. - Arthroscopic view of torn posterior horn of lateral meniscus (PHLM) associated with posterolateral plateau fracture.

after arthroscopic articular depression reduction and preliminary raft screws fixation (figure 3 ).

After anesthesia, patients were placed in a supine position on a radiolucent orthopedic table. With formal sterilization and draping of the affected limb, the calf was exposed to monitor edema and increased compartment pressure during arthroscopy. The contralateral iliac bone graft site was prepared to be harvested in cases where large bone defects were found. Tourniquet was inflated $150 \mathrm{mmHg}$ above systolic pressure with antibiotic administration prior to its inflation.

Knee arthroscopy was initially performed for assessment of fracture lines, locations of articular surface depression and associated meniscal/ligamentous injuries. It was performed in all isolated posterolateral fractures however it wasn't the rule in all cases of the combined fractures. Pump pressure was controlled not to be over $40 \mathrm{mmHg}$ and an outflow superolateral portal was used. The joint cavity was washed removing blood clots and partial synovectomy was done to clearly visualize the amount of depression and identify any meniscal tears (figure 4).

After orienting with the depressed area in the posterolateral column, with the knee flexed not more than $90^{\circ}$ and supported in varus position to

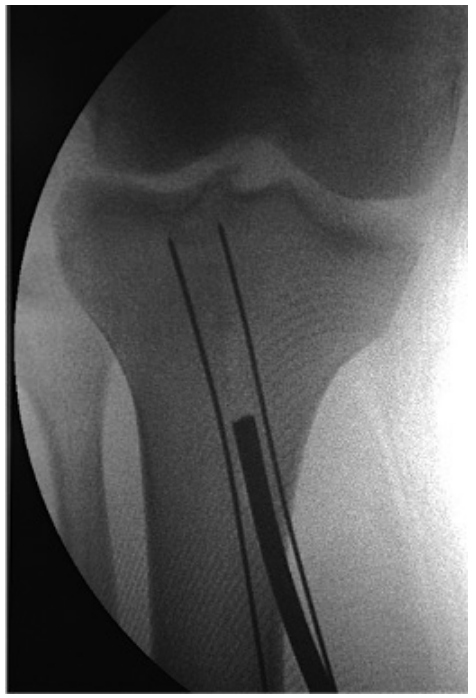

Fig 5. - The railway creation with bone impactor for elevation of depressed plateau fracture.

widen the lateral joint space, an anterior cruciate ligament (ACL) guide system was used to insert two guide wires from the anteromedial proximal tibia $10 \mathrm{~cm}$ from joint line towards the edge of the depressed plateau to create a railway for the bone graft impactor which was inserted after creating a bone window in the anteromedial cortex (figure 5). In cases with associated anteromedial column fractures, the already present split in the medial cortex was used as a window for passage of the impactor. After adequate elevation of the depression via the impactor was confirmed arthroscopically, a guide wire for rafting screw was inserted from lateral to medial to prevent collapse. In cases with comminuted depressed surface (plateau islands) this step was repeated. The number and size of the rafting screws were decided according to the surface area and degree of comminution. Rafting screws fixation was followed by reassessment of the joint space without varus support to confirm complete elevation of the depressed fragment(s) (figure 6).

Lateral meniscus tears were sutured with allinside meniscus implant Cinch ${ }^{\circledR}$ (Arthrex, Naples, FL). Bony avulsions of ACL ligament from tibia were transfixed into bone via no.5 Fiberwire ${ }^{\circledR}$ (Arthrex, Naples, FL) over bony tunnels in the anteromedial cortex. Medial meniscus tears were 


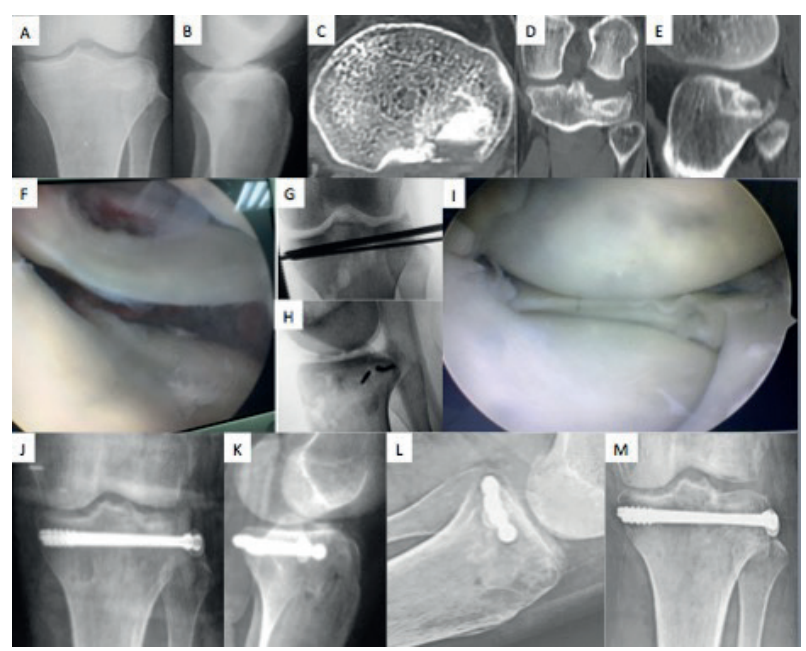

Fig 6. - Isolated posterolateral tibial plateau fracture (A, B) : preoperative X-rays with double line sign $(\mathrm{C}, \mathrm{D}, \mathrm{E})$ : CT scan of split-depressed posterolateral fracture with fracture segment $<15 \mathrm{~mm}$ in height, extension of the articular part of the split segment $<15 \mathrm{~mm}$, minimal comminution $<3(\mathrm{~F})$ : Arthroscopic view showing the widened lateral joined space due to depression $(\mathrm{G}, \mathrm{H})$ : Reduction of depression and temporary fixation by K-wires (I) : Meniscal repair and check of depressed segment elevation with narrowing of joint space "drive-through sign" $(\mathrm{J}, \mathrm{K})$ : Immediate postoperative X-rays $(\mathrm{L}, \mathrm{M})$ : 14 weeks postoperative X-rays after fracture union.

sutured with outside-inside technique if needed. Small radial or flap tears were excised.

Image intensifier was used to double check reduction following raft screws fixation and when patients were repositioned prone for additional procedures. Fixation was completed whether with a buttress plating for posterolateral column in prone position according to protocol, or other columns in the sequence of anteromedial first (corner stone of plateau) followed by the arthroscopic assisted depression fixation, posteromedial column, posterolateral buttressing, and lastly the anterolateral column (figures 6,7).

Images were also obtained after final bony fixation to assess restoration of both coronal and sagittal alignments as well as the articular surface. Examination for medial and posterolateral ligament complex laxity after bone fixation was done. Management of any present laxity was delayed to allow early range of motion rehabilitation and give a chance for spontaneous healing of medial collateral ligament. Only when posterolateral approach was

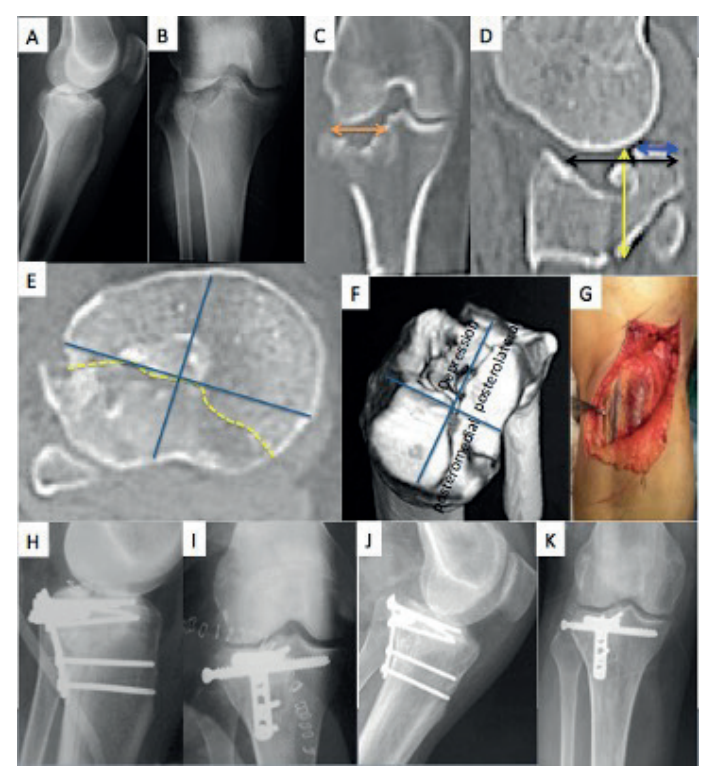

Fig 7. - Associated posterolateral and posteromedial tibial plateau fracture $(\mathrm{A}, \mathrm{B})$ : preoperative X-rays $(\mathrm{C}, \mathrm{D})$ : $\mathrm{CT}$ scan of split-depressed posterolateral fracture with fracture segment $>15 \mathrm{~mm}$ in height [yellow arrow] and width [orange arrow], extension of the articular part of the split segment $<15 \mathrm{~mm}$ [blue arrow], minimal comminution $<3$, but extent of depression in tibial plateau $>1 / 3$ [black arrow] (E) CT scan axial view showing involvement of posteromedial column $(\mathrm{F})$ : 3D-CT scan showing involvement of posteromedial column (G) : According to protocol after arthroscopic reduction of depression, posterior approach was done for posterior buttress plating $(\mathrm{H}, \mathrm{I})$ : Immediate postoperative $\mathrm{X}$-rays $(\mathrm{J}, \mathrm{K}): 2$ years postoperative X-rays after fracture union. Benchmarks which are used for dividing the plateau surface into 4 columns are : Antero-Posterior line : from medial third of tibial tuberosity till PCL insertion (posterior tibial crease). Medio-Lateral line : from posterior border of superficial MCL attachment till a point anterior to superior tibio-fibular joint (anterior cortex of fibula).

used, posterolateral corner injuries were addressed early.

X-rays follow up was done on second day postoperative, at three weeks, six weeks and then monthly till fracture union. When there was a double-line sign in the postoperative or follow up plateau view X-rays which raised suspicion for inaccurate reduction or re-depression, CT scan was performed. The minimum follow up period was 24 months (average 26 months). No patients were lost till the last follow up.

Rehabilitation program under supervision of physiotherapy and rehabilitation unit started from the 
second day with quadriceps muscle strengthening, anti-inflammatory anti-edema measures, nonweight bearing mobilization with crutches till six weeks after which gradual assisted weight bearing progressed till full fracture union, knee range of motion limited to $30^{\circ}$ in first three weeks, increased gradually to $90^{\circ}$ at six weeks, after which the range of motion was progressed to obtain full range within the next six weeks.

Patients were evaluated regarding radiological union time, range of motion, KOOS score and presence of any complications.

\section{RESULTS}

From the forty-two patients included in this study, thirty-three were males and only nine patients were females. The mean age of the patients was thirty-six ranging from (22-59). As regard the mechanism of injury, twenty-six fractures were due to high-energy mechanisms while sixteen were due to low energy mechanisms with a statistically significant difference between the isolated and associated posterolateral plateau fractures with a higher incidence of associated plateau injuries in high energy trauma.
The overall split posterolateral tibial plateau fracture subtype was nine patients of which 3 patients needed buttress plating while the rest could be fixed with rafting screws only (these six fractures were fulfilling the proposed criteria for screw only fixation in split fractures). No bone grafting was done for such a fracture whether when isolated or in association with other columns fracture.

For the depressed subtype, 19 patients were included. Plating was done for 6 patients from extended anterolateral approach while 13 patients were fixed with screws. Bone grafting was done in 15 patients. The 4 patients without grafting had a pre-reduction depression of less than $5 \mathrm{~mm}$ so screws were enough to fill the defect. This was statistically significant as regard the need of bone grafting in depressed fractures to add stability for fixation with screws only specifically with ASIF techniques to fill the reduction pathway.

Fourteen patients had a split-depressed subtype with statistically significant occurrence in association with other columns fracture. 10 patients were fixed with plate while 4 had been fixed with screws only. Bone grafting was done in three patients. On the contrary of depressed only fractures, bone grafting in split-depressed fractures was not necessary and

Table 2. - Different fixation methods and the need for bone graft

\begin{tabular}{|c|c|c|c|c|c|}
\hline \multicolumn{2}{|c|}{ Split subtype } & \multicolumn{2}{|c|}{ Split depressed } & \multicolumn{2}{|c|}{ Depressed } \\
\hline \multicolumn{2}{|c|}{9} & \multicolumn{2}{|c|}{14} & \multicolumn{2}{|c|}{19} \\
\hline Raft screws & Plating & Raft screws & Plating & Raft screws & Plating \\
\hline 6 & 3 & 4 & 10 & 13 & 6 \\
\hline \multicolumn{2}{|c|}{$\begin{array}{c}\mathrm{X}^{2}=1.000 \\
\mathrm{p}=0.317\end{array}$} & \multicolumn{2}{|c|}{$\begin{array}{c}\mathrm{X}^{2}=2.571 \\
\mathrm{p}=0.109\end{array}$} & \multicolumn{2}{|c|}{$\begin{array}{c}\mathrm{X}^{2}=2.579 \\
\mathrm{p}=0.108\end{array}$} \\
\hline Bone graft & No graft & Bone graft & No graft & Bone graft & No graft \\
\hline- & 9 & 3 & 11 & 15 & 4 \\
\hline \multicolumn{2}{|c|}{............... } & \multicolumn{2}{|c|}{$\begin{array}{l}X^{2}=4.571 \\
p=0.033^{*}\end{array}$} & \multicolumn{2}{|c|}{$\begin{array}{l}X^{2}=6.368 \\
p=0.012^{*}\end{array}$} \\
\hline
\end{tabular}

$* \mathrm{p}<0.05$ is significant.

Table 3. - The KOOS score and average time of union according to the fracture classification

\begin{tabular}{|l|c|c|c|c|c|}
\hline Parameters & \multicolumn{3}{|c|}{ Facture type } & \multirow{2}{*}{ p value } & Significance \\
\cline { 2 - 5 } & $\begin{array}{c}\text { Split } \\
\mathbf{n = 9} \\
(\mathbf{M e a n} \pm \text { SD) }\end{array}$ & $\begin{array}{c}\text { Split depressed } \\
\mathbf{n = 1 4} \\
(\text { Mean } \pm \text { SD) }\end{array}$ & $\begin{array}{c}\text { Depressed } \\
\mathbf{n = 1 9} \\
(\text { Mean } \pm \text { SD) }\end{array}$ & & NS \\
\hline KOOS & $82.33 \pm 2.83$ & $80.64 \pm 4.73$ & $81.16 \pm 2.63$ & 0.530 & NS \\
\hline Time of union & $16.00 \pm 2.45$ & $16.71 \pm 2.43$ & $16.10 \pm 2.60$ & 0.735 & \\
\hline
\end{tabular}

$\mathrm{NS}=$ non-significant. 
Table 4. - KOOS score and average time of union whether fracture is isolated or combined

\begin{tabular}{|l|c|c|c|c|}
\hline Parameters & \multicolumn{2}{|c|}{ Fracture type } & \multirow{2}{*}{ S value } & Significance \\
\hline KOOS & $\begin{array}{c}\text { Isolated } \\
\mathbf{n = 1 1} \\
(\mathbf{M e a n} \pm \text { SD) }\end{array}$ & $\begin{array}{c}\text { Combined } \\
\mathbf{n = 3 1} \\
(\mathbf{M e a n} \pm \text { SD) }\end{array}$ & p & NS \\
\hline Time of union & $82.00 \pm 3.61$ & $80.96 \pm 3.45$ & 0.404 & NS \\
\hline
\end{tabular}

$\mathrm{NS}=$ non-significant.

Table 5. - Patients' data with loss of reduction after fixation

\begin{tabular}{|c|c|c|c|c|c|c|c|c|c|}
\hline & Fracture type & Isolated/combined & Gender & Age & $\begin{array}{l}\text { Mechanism of } \\
\text { injury }\end{array}$ & $\begin{array}{l}\text { Method of } \\
\text { fixation }\end{array}$ & $\begin{array}{l}\text { Application } \\
\text { of bone graft }\end{array}$ & KOOS & $\begin{array}{c}\text { Time to } \\
\text { union }\end{array}$ \\
\hline 1 & Split depressed & Combined & Male & 45 & Slippage & $\begin{array}{l}\text { Rafting } \\
\text { screws }\end{array}$ & & 83 & 17 \\
\hline 2 & Depressed & Combined & Male & 25 & Road traffic & $\begin{array}{c}\text { Buttress } \\
\text { plate }\end{array}$ & Applied & 82 & 18 \\
\hline 3 & Depressed & Combined & Female & 31 & $\begin{array}{c}\text { Hit by heavy } \\
\text { object }\end{array}$ & $\begin{array}{l}\text { Buttress } \\
\text { plate }\end{array}$ & Applied & 80 & 14 \\
\hline 4 & Split depressed & Combined & Male & 25 & Road traffic & $\begin{array}{c}\text { Buttress } \\
\text { plate }\end{array}$ & & 74 & 15 \\
\hline 5 & Split depressed & Combined & Male & 29 & Road traffic & $\begin{array}{l}\text { Buttress } \\
\text { plate }\end{array}$ & & 72 & 18 \\
\hline
\end{tabular}

this was also statistically significant but for the favor of no bone grafting. (Table 2)

All the patients had been followed for a minimum of 24 months (average of 26 months). 16 patients had lateral meniscus tears that were sutured with all inside technique, 3 with avulsed ACL that were repaired with transosseous sutures over bony tunnels and 3 had medial meniscus tears with only 1 case that needed repair while the other 2 cases had small radial tears that were excised. Fracture union was evidenced at a mean of 16.3 (12-22) weeks with no additional bone grafting. The mean KOOS score was $81(72-88)$ at the last follow up. The average knee range of motion was $\left(0^{\circ}-127^{\circ}\right)$ with an average loss of $8^{\circ}$ degrees of flexion in comparison to contralateral knee. There was no statistical significant difference as regard the different three types of posterolateral column fractures in terms of functional and radiological results whether occurring alone or in combination with other columns fracture (Tables $3 \& 4$ ).

Comparing isolated and associated posterolateral column fractures, there was no statistical significant difference as regard the average time of union and function (Tables $3 \& 4$ ).
No compartment syndrome was recorded in any of the cases. Single case of temporary common peroneal nerve palsy occurred where a posterolateral approach was used that was improved after 4 months with complete regain of function. 5 patients had loss of reduction in the form of depression (2-5 mm) that did not need further intervention in this short term follow up, no special complaint as regard the pain nor the function were recorded (table 5). There was no evidence of loss of the coronal or the sagittal alignment at last follow up. One patient had deep infection (in which posteromedial and extended anterolateral approaches were done) where serial debridement was done with retained implants and negative pressure wound therapy was used after eradication of infection. Two patients had superficial infection treated with extended antibiotics with no need for surgery. The patients returned to work in an average of 4 months postoperatively. Participation in recreational sports activities started after 8 months.

\section{DISCUSSION}

Many classification systems had been described for tibial plateau fractures but none is ideal as regard 
the inter-observer reliability and the prediction of postoperative outcome. The inter-observer reliability increases with CT scan based classifications (1721). An update of the three-column theory into four columns had been suggested dividing the posterior column into posteromedial and posterolateral columns (22-24).

In the past few years, different parameters for mapping of plateau fractures and descriptive criteria for the morphological characteristics of the posterolateral fragment fractures (PLF) were illustrated including its height, angle and surface area (24-25). To our knowledge none linked these different morphological characteristics to a clinical guide to choose the best surgical approach and fixation implant.

PLF gained attention because of difficult approaches and reduction techniques. Although many surgical approaches are available for posterolateral fragment, none is ideal in providing maximum visualization and easy reduction and application of fixation implant that provides best mechanical stability (26-28).

In our series the incidence of PLF (whether isolated or combined with other columns) was $34.4 \%$. Which is comparable to other published studies in literature $(15 \%-44.2 \%)$. This incidence increased in comparison to that previously thought because of the use of CT scan $(21,24,25)$.

Surgical approaches for PLF can be divided into posterior, posteromedial and posterolateral with or without fibular head osteotomy. These approaches allow direct reduction of the fracture fragment but with high risk of injury to vital neurovascular structures including the popliteal neurovascular structures, common peroneal nerve, inferior lateral geniculate artery and anterior tibial vessels that cross the interosseous ligament from posterior to anterior about five centimeters from the joint line limiting the distal extension of these approaches. Associated anterior columns fractures cannot be accessed from these approaches and usually requires changing of patient position from prone to supine which prolong the operative time. Different modifications of the posterolateral approaches were described. Unfortunately, there is no specific criteria upon which surgeon can choose the approach in different PLF modalities that has the least harmful risk $(8,11,12,29-33)$.

The extended anterolateral approach described by Cho JW et al. (34) allows good visualization of the PLF in the supine position. Combined anterolateral column fracture can be managed from the same approach. Other columns can be also managed without changing patient position. This approach carries the disadvantage of uneasy placement of posterior buttress plate that gives better biomechanical stability specifically in split and split-depressed subtypes.

Chen HW et al. (35) proposed a treatment guide for posterior tibial plateau fractures based on different five types including both posteromedial and posterolateral fragments. This classification was depending on retrospective analysis of 39 cases that all had been approached from posterior, in contrast to this study, they ignored the presence of associated other columns fractures and did not link the fixation implant with the fracture pattern.

Cho JW et al. (34) proposed a treatment algorithm for posterolateral fractures either as isolated or associated with other columns that was very beneficial. They were dependent on two approaches ; extended anterolateral and the posterolateral approaches ; they dealt with the posterolateral fracture as if it is all the same and did not explain the different morphological patterns as depressed, split, or split depressed and their influence on the choice of the approach and the fixation implant. They preferred the use of the newly introduced rim plates. In the current study the morphological pattern of the fracture was identified and the management was applied according to the algorithm in (figure 2).

Meniscal and ligamentous injuries mainly lateral meniscal tears and ACL disruption affect postoperative functional outcome and need to be addressed to accelerate rehabilitation programs. ARIF showed good to excellent long-term outcomes with more precise diagnoses, and provide safe management when combined with the biomechanically suitable implant $(15,16,36)$. This goes hand in hand in the current study as arthroscopic examination was done to all the patients and ideal soft tissue management was done to improve the long term outcome for the patients. 
From the previously mentioned problems the need for a treatment algorithm for management of tibial plateau fractures urge especially with PLF either isolated or combined. The authors developed this treatment protocol trying to guide surgeons when faced with tibial plateau fractures with PLF either isolated or in association with other column injuries. "Do no further harm" was the message from this algorithm in order to choose the least traumatic approach that provide the appropriate visualization for reduction and allow for fixation that provide stability till fracture union.

The morphological characteristics of the posterolateral fragment were based on the work of Sohn HS et al. (26). In cases of split and split-depressed fracture subtypes, the choice of fragment size (extent of articular surface in the split fragment) either more than or less than $1.5 \mathrm{~cm}$ was based on morphological criteria found in literature describing the average surface area of PLF of about $14 \pm 6 \%$ of the plateau. Together with putting in consideration that fragments height (cranio-caudal dimension) less than $1.5 \mathrm{~cm}$ means that it is within the flare of the posterolateral cortex, so plating of these fragments might not be necessary and applying a buttress plate will require a skewed contouring of the plate which is difficult and might annoy the patient flexion range and rub on the soft tissue.

Again for the width (medio-lateral dimension) means that it represents a small surface area for the transverse limb in the T-plate, otherwise using a one third tubular or straight plate will lead to fixation of the fragment with only one screw, so again plating might not be necessary (25). That was comparable to our detailed algorithm in the current study.

For multifragmentary comminuted articular surface we aimed at the best rafting technique through plating that could maintain stability of the fragments till union together with or without bone grafting (37).

Therefore, in cases of isolated PLF, small sized split fragments with height, width and extent less than $1.5 \mathrm{~cm}$ or those split depressed with less than three fragments not extending towards the anterolateral column $(<1 / 3$ plateau anteroposterior dimensions) can be fixed with rafting screws only and this can be achieved through ARIF that is done in supine position and can address the associated soft tissue injuries. Larger fragments will need to be buttressed with a plate that can be approached through a posterolateral approach after performing arthroscopic assessment and meniscal and ligamentous injuries management with exchange of patient position from supine to prone that facilitate reduction of the fracture. Comminuted fractures and those with a depression $>1 / 3$ tibial plateau can be buttressed from lateral or posterior with the use of rim plates through an extended anterolateral approach with or without posteroanterior rafting screws.

For depressed fractures, the comminution and the extent into the plateau are the main parameters determining the fixation implant. And they can be dealt with as in split-depressed fractures.

In cases of associated columns fractures, we started our reduction from anteromedial column that is the cornerstone upon which building up our plateau can be achieved in a manner of anticlockwise rotation. Posteromedial and posterolateral columns, posterior approach was used. Anterolateral and posterolateral columns, extended anterolateral approach was used. Anteromedial and posterolateral columns, medial MIPPO (minimally invasive percutaneous plate osteosynthesis) together with treating the posterolateral as if isolated. And if more than two columns affected. Posteromedial approach together with extended anterolateral approaches were the gates to deal with the whole circumference of the plateau. This goes with many studies published in the literature supporting such a protocol $(1,6,7,9,12,27,34,35)$.

In a series of seven cases done by Cho JW et al. (34) the union of fracture was achieved in all the cases at a mean of 12 weeks after surgery (818 weeks) with no loss of reduction in their follow up and the patients reported functional outcomes using the Lysholm score (average, 88.7 ; range, 7295) with no evidence of complications related to their approach. That was comparable to our cohort except in the presence of complications as 1 of our patients developed temporary common peroneal nerve palsy that was completely improved after 4 months post-surgery. Also, 1 patient had deep infection (in which posteromedial and extended anterolateral approaches were done) where serial 
debridement was done with retained implants and negative pressure wound therapy was used after eradication of infection. 2 patients had superficial infection treated with extended antibiotics with no need for surgery. This increased incidence of complications was attributed to the relatively higher number of patients in our series (42) in comparison to the 7 cases in the other study.

In a prospective cohort study of 287 patients done by Wang Y et al. (2), the mean time to union was 13.5 weeks (range : 10-28), average functional knee society score was 93.0 (range : 80-95). The average range of motion of the fractured knees was $1.5-121.5^{\circ}$. Twelve patients suffered from superficial skin infection, one limited skin necrosis and two developed wound dehiscence that all healed without operative management. These results were comparable to our series. 2 patients of their study had intra operative vascular injury repaired primarily and 13 cases (4.5\%) had temporarily common peroneal nerve injury compared to our series $(2.4 \%)$ and none of our patients developed vascular injury.

The main limitation of this study is the small number of patients especially in the isolated postero-lateral fragment group (11 patients) and the short term follow up which could not detect the development of posttraumatic arthritis taking in consideration that 5 patients had loss of reduction accuracy in the form of depression of equal or less than $5 \mathrm{~mm}$. They had no special complaint as regard pain or function. Actually this might not progress into arthritis especially after repair of lateral meniscal tear that protects the articular surface.

The suggested protocol may provide a clue to solve the enigma of planning a posterolateral tibial plateau fracture whether isolated or associated with other columns fractures as regard the proper approaches and fixation implants depending on proper diagnosis based on the CT four column classification without adding further harm to the patient.

\section{REFERENCES}

1. Prat-Fabregat S, Camacho-Carrasco P. Treatment strategy for tibial plateau fractures : an update. EFORT Open. Rev. 2017 ; 1 : 225-232.
2. Wang Y, Luo CF, Zhu Y, et al. Updated Three-Column Concept in surgical treatment for tibial plateau fractures A prospective cohort study of 287 patients. Injury. 2016 ; 47(7) : 1488-1496.

3. Luo CF, Sun H, Zhang B, et al. Three-column fixation for complex tibial plateau fractures. J. Orthop Trauma. 2010 ; $24: 683-92$

4. Bhattacharyya T, McCarty LP 3rd, Harris MB, et al. The posterior shearing tibial plateau fracture: treatment and results via a posterior approach. J Orthop. Trauma. $2005 ; 19: 305-310$.

5. Kim CW, Lee CR, An KC, et al. Predictors of reduction loss in tibial plateau fracture surgery : Focusing on posterior coronal fractures. Injury. 2016 ; 47 : 1483-1487.

6. Kokkalis ZT, Iliopoulos ID, Pantazis C. What's new in the management of complex tibial plateau fractures? Injury. $2016 ; 47: 1162-1169$.

7. Weaver MJ, Harris MB, Strom AC, et al. Fracture pattern and fixation type related to loss of reduction in bicondylar tibial plateau fractures. Injury. $2012 ; 43(6)$ : 864-9.

8. Chen HW, Liu GD, Ou S, et al. Open reduction and internal fixation of posterolateral tibial plateau fractures through fibula osteotomy-free posterolateral approach. $J$. Orthop. Trauma. 2014, 28(9) : 513-7.

9. Musahl V, Tarkin I, Kobbe P, et al. New trends and techniques in open reduction and internal fixation of fractures of the tibial plateau. J. Bone Joint Surg. Br. 2009 ; 91 : 426-433.

10. Stannard JP, Brown SL, Robinson JT, et al. Reconstruction of the postero-lateral corner of the knee. Arthroscopy. $2005 ; 21: 1051-1059$.

11. Tao J, Hang DH, Wang QG, et al. The posterolateral shearing tibial plateau fracture : treatment and results via a modified posterolateral approach. Knee. 2008 ; 15 : 473479.

12. Carlson DA. Posterior bicondylar tibial plateau fractures. J. Orthop. Trauma. $2005 ; 19: 73-78$.

13. Frosch KH, Balcarek P, Walde T, et al. A new posterolateral approach without fibula osteotomy for the treatment of tibial plateau fractures. J. Orthop. Trauma. $2010 ; 24(8)$ : 515-20.

14. Yi-Sheng Chan, Chih-Hao, Yang-Pin Lo, et al. Arthroscopy-Assisted Surgery for Tibial Plateau Fractures : 2- to 10-Year Follow-up Results. Arthroscopy. 2008 ; 24(7) : 760-768.

15. Elabjer E, Benčić I, Ćuti T, et al. Tibial plateau fracture management: arthroscopically-assisted versus ORIF procedure - clinical and radiological comparison. Injury. 2017 ; 48 Suppl 5 : S61-S64.

16. Hartigan DE, McCarthy MA, Krych AJ, et al. Arthroscopic-assisted reduction and percutaneous fixation of tibial plateau fractures. Arthrosc. Tech. $2015 ; 2 ; 4(1)$ : e51-5.

17. Chang SM, Hu SJ, Du SC, et al. Four-quadrant/column classification of tibial plateau fractures. Int. Orthop. 2018 ; 42(3) : 725-727. 
18. Maripuri SN, Rao P, Manoj-Thomas A, et al. The classification systems for tibial plateau fractures: how reliable are they? Injury. 2008 ; 39(10) : 1216-21.

19. Millar SC, Arnold JB, Thewlis D, et al. A systematic literature review of tibial plateau fractures: What classifications are used and how reliable and useful are they? Injury. 2018 ; 49(3) : 473-490.

20. Taşkesen A, Demirkale İ, Okkaoğlu MC, et al. Intraobserver and interobserver reliability assessment of tibial plateau fracture classification systems. Eklem Hastalik Cerrahisi. 2017 ; 28(3) : 177-81.

21. Hu YL, Ye FG, Ji AY, et al. Three-dimensional computed tomography imaging increases the reliability of classification systems for tibial plateau fractures. Injury. 2009 ; 40(12) : 1282-5.

22. Krause M, Frosch KH. Response to the letter-to-the-editor by Dhillon et al. "Simple four column classification can dictate treatment for intra articular tibial plateau fractures much better than ten segment classification", Injury. 2017 ; 48(10) : 2369-2370.

23. Martínez-Rondanelli A, Escobar-González SS, HenaoAlzate A, et al. Reliability of a four-column classification for tibial plateau fractures. Int. Orthop. 2017 ; 41(9) : 18811886.

24. Krause M, Menzdorf L, Preiss A, et al. Are there four tibial plateau columns? Yes there are, as illustrated by a postero-lateral apple-bite fracture. Response to a letter-tothe-editor. Int. Orthop. 2018 ; 42(2) : 443-446.

25. Xiang G, Zhi-Jun P, Qiang Z, et al. Morphological characteristics of posterolateral articular fragments in tibial plateau fractures. Orthopedics. $2013 ; 1 ; 36(10)$ : e125661.

26. Sohn HS, Yoon YC, Cho JW, et al. Incidence and fracture morphology of posterolateral fragments in lateral and bicondylar tibial plateau fractures. J. Orthop. Trauma. $2015 ; 29(2)$ : 91-7.

27. Zhang W, Luo CF, Putnis S, et al. Biomechanical analysis of four different fixations for the posterolateral shearing tibial plateau fracture. Knee. $2012 ; 19(2): 94-8$.
28. Musahl V, Tarkin I, Kobbe $\mathbf{P}$, et al. New trends and techniques in open reduction and internal fixation of fractures of the tibial plateau. J. Bone Joint Surg. Br. 2009 ; $91: 426-433$.

29. Stannard JP, Brown SL, Robinson JT, et al. Reconstruction of the postero-lateral corner of the knee. Arthroscopy. $2005 ; 21: 1051-1059$.

30. Yu GR, Xia J, Zhou JQ, et al. Low-energy fracture of posterolateral tibial plateau : treatment by a posterolateral prone approach. J. Trauma Acute Care Surg. 2012; 72 : 1416-1423.

31. Sun H, Luo CF, Yang G, et al. Anatomical evaluation of the modified posterolateral approach for posterolateral tibial plateau fracture. Eur. J. Orthop. Surg. Traumatol. $2013 ; 23: 809-818$.

32. Huang YG, Chang SM. The posterolateral approach for plating tibial plateau fractures: problems in secondary hardware removal. Arch. Orthop. Trauma Surg. 2012; $132: 733-734$.

33. Chang SM, Zheng HP, Li HF, et al. Treatment of isolated posterior coronal fracture of the lateral tibial plateau through posterolateral approach for direct exposure and buttress plate fixation. Arch. Orthop. Trauma Surg. 2009 ; 129 : 955-962.

34. Cho JW, Samal P, Jeon YS, et al. Rim Plating of Posterolateral Fracture Fragments (PLFs) Through a Modified Anterolateral Approach in Tibial Plateau Fractures. J. Orthop. Trauma. 2016 ; 30 : e362-e368.

35. Chen HW, Chen CQ, Yi XH. Posterior tibial plateau fracture : a new treatment-oriented classification and surgical management. Int. J. Clin. Exp. Med. 2015 ; 8(1) : 4729.

36. Warner SJ, Garner MR, Schottel PC, et al. The Effect of Soft Tissue Injuries on Clinical Outcomes After Tibial Plateau Fracture Fixation. J. Orthop. Trauma. 2018 ; 32(3) : 141-147.

37. Sun H, Zhu Y, He QF, et al. Reinforcement strategy for lateral rafting plate fixation in posterolateral column fractures of the tibial plateau : The magic screw technique. Injury. 2017 ; 48(12) : 2814-2826. 